\title{
Towards a reduced chemistry module of a He-Ar-Cu hollow cathode discharge
}

\author{
D Mihailova ${ }^{1}, \mathrm{~J}$ van Dijk ${ }^{1}, \mathrm{M} \mathrm{Grozeva}^{2}, \mathrm{G} \mathrm{Degrez}^{3}$ and \\ J J A M van der Mullen ${ }^{1}$ \\ ${ }^{1}$ Department of Applied Physics, Eindhoven University of Technology, \\ P.O. Box 513, 5600 MB, Eindhoven, The Netherlands. \\ 2 Institute of Solid State Physics, Bulgarian Academy of Sciences, \\ 72 Tzarigradsko Chaussee, 1784 Bulgaria \\ ${ }^{3}$ Service de Mécanique des Fluides, Université Libre de Bruxelles, \\ 50 avenue F.D. Roosevelt, 1050 Bruxelles, Belgium \\ E-mail: diana@epgmod.phys.tue.nl
}

\begin{abstract}
This study is aimed to find a reduced chemistry module for hollow cathode discharge excited in a He-Ar-Cu mixture. This enables to construct lean and realible models that can be used as a part of the design tool of hollow cathode discharges (HCDs). To this end estimative calculations and numerical simulations are performed at optimal conditions for lasing. An analysis of the species behaviour and reactions is made and as a result the model is simplified by means of reducing the number of species and reactions. The consequences of these reductions is justified by comparing the results of the simplified models with those of a more complete one. This study delivers a model that is chemical lean and thus, much less time consuming. It can be used in optimization studies to find the optimum in the plasma control parameter-set of HCDs. The technique developed in this study for HCDs can be applied to glow discharges in general.
\end{abstract}




\section{Introduction}

The interest in complex chemistry plasmas is increasing rapidly during the last decade. This is among others boosted by the booming attention paid to plasmas for biomedical applications and environmental technology. In all these modern applications the classical atomic plasma composed of one single noble gas species is replaced by plasmas created in rich chemical fillings. As a consequence of plasma activities these filling are transformed into a myriad of excited atoms, molecules, radicals and various types of ions. This gives firm ground to the statement that the problem of plasma physics nowadays mostly lies in plasma chemistry.

One of the serious problems is manifested by the increase in the number $N$ of relevant species. For a complex plasma this can easily go beyond $N=100$. Even more seriously is the number of conversion reactions between these species for which we can expect a scaling with something like $N^{2}$. A further complicating factor is the large variety in reaction speeds. Here we can easily find differences in the order of $10^{3}$. This makes that complex chemistry plasma models can become extremely stiff.

In search for the most appropriate strategy to model these plasmas the question rises whether it is really needed to treat all the species individually. Should we compute for all the species their densities and transport fluxes? Or, do there exist systematic methods that can lead to a reduced chemistry? A reduced chemistry module is a module that is small enough to make the grand plasma model lean while the computation of the main plasma properties is not in jeopardy.

This study is devoted to the search of a reduced chemistry module for hollow cathode discharges (HCDs) used for laser applications. Such a module enables to construct a lean and reliable model that can be used as part of a design tool of HCD lasers. The aim of this tool is to find, by modelling, the optimum in the plasma control parameters-set of these plasmas. Since the control parameter space is enormous it is requested that the model converges easily so that the exploration of this hyper-space is not too much time consuming. With this chemical lean model it is then possible to find the best version of the geometrical construction, the anode-cathode patterns, the current settings, the fill-chemistry, etc.

This study starts with a model that describes a longitudinal sputtering HCD used for laser application. It has a cylindrical geometry with two anodes at both sides as described in detail in $[1,2]$. The hollow cathode metal cylinder is with a length of $50 \mathrm{~mm}$ and an inner diameter of $4 \mathrm{~mm}$. The sputtered cathode material is $\mathrm{Cu}$ and the operating gas is a mixture of He with $5 \%$ of Ar. The total gas pressure equals $2.3 \mathrm{kPa}$ and the discharge is excited by a $3 \mathrm{~ms}$ current pulse.

The model used for this study is the time-dependent fluid model MD2D described in detail in [2]. The simulation model is based on a set of 10 species and 23 reactions. For these active species the balance equations are solved each of them describing the competition between the transport and chemistry on a per-species base. The results of the simulation includes the spatial and temporal evolution of densities, the reaction rates and transport fluxes of the relevant species. These are used to get volume-averaged values of these quantities. From this output a detailed analysis of the contribution of production/destruction and transport for each species can be obtained so that the most important species and the relevant conversion channels can be determined. The insight obtained can subsequently be used to construct a grand model based on a reduced chemistry. To justify the possible consequences of the chemistry reduction we will compare the results of the simplified models with those of the initial more complex chemistry model. These steps will allow to define the validity range of this reduction method and its applicability to other plasma sources.

The paper is organised as follows: in section 2 the hierarchy in processes is explored and estimative calculations are made. Section 3 describes the set of species and reactions used in the numerical model. A detailed analysis is made and presented in section 4 Based on this analysis simplified models are constructed and the comparison with the more complete model is presented in subsections. Finally, section 5 summarises the observations. 


\section{Hierarchy in processes}

The analysis of the hierarchy of chemical species and conversion processes will be guided by the general form of the particle balance showing the competition between chemistry (production and destruction processes) and transport. For the particle $p$ it reads:

$$
\frac{\partial n_{p}}{\partial t}+\nabla \cdot \boldsymbol{\Gamma}_{p}=S_{p}
$$

where $\boldsymbol{\Gamma}_{p}$ is the flux density and $S_{p}$ is net source term. The time-derivative can be omitted since the duration of the excitation current pulse, being $3 \mathrm{~ms}$, greatly exceeds the time-scales of the plasma processes. This implies that steady state (SS) is obtained during the pulse. Omitting the transient term, the continuity equation reads:

$$
\nabla \cdot \boldsymbol{\Gamma}_{p}=S_{p}
$$

We start with an exploration of the left hand side, the transport side, of this equation. After that the right hand side will be dealt with.

By writing the transport term as $\nabla \cdot \boldsymbol{\Gamma}_{p}=n_{p} \nu_{p}{ }^{t}$, we introduce the transport frequency $\nu_{p}{ }^{t}$ of species $p$. The flux density $\boldsymbol{\Gamma}_{p}$ is given by:

$$
\boldsymbol{\Gamma}_{p}=\mu_{p} \mathbf{E} n_{p}-D_{p} \nabla n_{p},
$$

where the mobility $\mu_{p}$ and diffusion coefficient $D_{p}$ of species $p$ are determined by collisions of these species with buffer gas atoms. We see that in general two driving forces are present: the drift generated by the electric field and the diffusion set up by gradients. It is clear that neutral particles are driven by the diffusion only. Due to the additional drift term, the ions will have higher velocities in general. We will come back to this later.

The right hand side of equation (2), the source term $S_{p}$ is determined by the reactions occurring in the discharge:

$$
S_{p}=\sum c_{p, r} R_{r}
$$

where $c_{p, r}$ is the net stoichiometric number of particles of species $p$ created in one reaction $r$ while $R_{r}$ is the corresponding reaction rate. The sum runs over all the reactions. The source term $S_{p}$ consists of positive $c_{p, r}>0$ (production) and negative $c_{p, r}<0$ (loss) contributions. So that it can be split in two terms - the production $\mathscr{P}_{p}$ and loss rate $\mathscr{L}_{p}$ :

$$
S_{p}=\mathscr{P}_{p}-\mathscr{L}_{p}
$$

Note that $\mathscr{P}_{p}$ is the total production rate: it is a sum over all the reactions in which $p$ is created. The same applies for the loss rate $\mathscr{L}_{p}$ : it is the total loss due to radiative and collision-induced transitions. The production rate can be written as:

$$
\mathscr{P}_{p}=\sum_{r} n_{q} \mathscr{D}_{q, p}
$$

where $\mathscr{D}_{q, p}$ is the frequency of the destruction processes of particle $q$ that lead to the production of $p$. The sum runs over all the reactions that lead to the production of $p$. The destruction of the same level $p$ is:

$$
\mathscr{L}_{p}=n_{p} \mathscr{D}_{p}
$$

where $\mathscr{D}_{p}=\sum_{r} \mathscr{D}_{p, r}$ is the destruction frequency of particle $p$, the so-called destruction factor of $p$. Thus, we can write the general form of the particle balance as:

$$
\begin{aligned}
& n_{p} \nu_{p}{ }^{t}=\mathscr{P}_{p}-n_{p} \mathscr{D}_{p} \\
& n_{p} \frac{\nu_{p}{ }^{t}}{\mathscr{D}_{p}}=\frac{\mathscr{P}_{p}}{\mathscr{D}_{p}}-n_{p} .
\end{aligned}
$$

In the case that the transport frequency $\nu_{p}{ }^{t}$ is much smaller than the destruction frequency, i.e. $\frac{\nu_{p}{ }^{t}}{\mathscr{D}_{p}} \ll 1$, equation (9) becomes:

$$
n_{p}=\frac{\mathscr{P}_{p}}{\mathscr{D}_{p}}
$$


So that the density of $p$ is completely determined by local reactions. The species will be designated by local chemistry (LC) species. Combining equations (10) and (7) leads to the criteria that holds for LC species:

$$
\frac{\mathscr{P}_{p}}{\mathscr{L}_{p}}=1
$$

The density of these LC species is generally much lower that that of the ground state atoms and ions. The latter, in general large density reservoirs, are apart from chemistry, also ruled by transport. These species are denoted by transport sensitive (TS) species. The buffer gas atoms in ground state are excluded, since they are assumed to be stationary in space and time.

The above shows that the dimensionless quantity $\nu_{p}^{t} / \mathscr{D}_{p}$ can be employed as a tool to distinguish between LC and TS species. For the last category the competition between transport and chemistry is important meaning that we have to solve the corresponding transport equations while for the first that is not needed and the continuity equation can be simplified. This ratio can be determined by running the grand model and to find $\nu_{p}^{t} / \mathscr{D}_{p}$ (or $\mathscr{P}_{p} / \mathscr{L}_{p}$ ) by post processing. Before doing so, we will perform some estimative calculations. We distinguish the following species:

- The buffer gas particles, the ground state atoms of He and Ar are assumed to be constant in time and space so they will not be equipped with a particle balance.

- For excited neutral species we can assume that the transport is purely diffusive and given by $\nu_{p}^{t}=D_{p} / R^{* 2}$, where $R^{*}$ is in the order of the radius of the discharge. For the diffusion coefficient $D_{p}$ we use the expression $D_{p}=k_{\mathrm{B}} T_{g} /\left(M_{p} \nu_{m}\right)$ with $\nu_{m}$ frequency of momentum transfer from the species to the buffer gas atoms, in this case He. We can use $\nu_{m}=n_{(H e)}\langle v\rangle \sigma$, where $\langle v\rangle=\left(k_{\mathrm{B}} T_{g} / M_{(H e)}\right)^{1 / 2}$ is the thermal velocity of the He atoms; this is justified since the relative velocity is mostly determined by thermal velocity of the lightest particle $(\mathrm{He})$. That means that the diffusion coefficient reads:

$$
D_{p}=\frac{\left(k_{\mathrm{B}} T_{g}\right)^{3 / 2} \sqrt{M_{(H e)}}}{M_{p}^{*} P \sigma m_{p}}=\frac{0.4}{M_{p}^{*}} \mathrm{~m}^{2} \mathrm{~s}^{-1},
$$

where $M_{p}^{*}$ is the mass number of the atom, $m_{p}$ the proton mass and $\sigma$ the cross section for momentum transfer. Inserting $T_{g}=1000 \mathrm{~K}, P=2.3 \mathrm{kPa}$ and $\sigma=10^{-19} \mathrm{~m}^{2}$, we get the value given at the right hand side. Of course the $\sigma$-value depends on the couple of interacting species but to get insight in the order of magnitude we took $\sigma=10^{-19} \mathrm{~m}^{2}$. Inserting for $R=2 \mathrm{~mm}$ we find for the $\mathrm{Cu}$ and $\mathrm{Ar}$ atoms values in the order of $\nu_{p}^{t}=2.10^{3} \mathrm{~s}^{-1}$. For excited He atoms this is more or less 40 times larger. For the excited atomic species we can use for the destruction frequency $\mathscr{D}_{p}=10^{7} \mathrm{~s}^{-1}$, which is taken equal to a typical value of the radiative decay frequency. So for excited species we get $\nu_{p}^{t} / \mathscr{D}_{p}$ values in the order of $10^{-3}$ to $10^{-2}$, meaning that they can be considered as being determined by local chemistry.

- For the excited ions we only have to consider $\mathrm{Cu}^{+*}$, which will represent the upper laser level. Apart from diffusion, this species will also be transported by drift. To get an estimate of the combination of drift and diffusion we can assume that the net effect leads to ambipolar diffusion. This implies a diffusion enhancement of the form $D_{a}=D_{p}\left(1+T_{e} / T_{h}\right)$, meaning that the transport frequency will be about 50 times higher. Inserting the radiative decay frequency in order of $10^{8} \mathrm{~s}^{-1}$ we find that $\nu_{p}^{t} / \mathscr{D}_{p}$ is in the order of $10^{-3}$. So that also the $\mathrm{Cu}^{+*}$ species are determined by local chemistry.

- For the atomic and molecular ions we have to compare the ambipolar diffusion with recombination processes. Several recombination channels have to be considered, which can be classified in 2-particle or 3-particle recombination channels. Due to the (global) charge neutrality the destruction of ions is closely related to that of electrons and as the plasma is strongly ionising we can expect that electrons are TS. So the same must apply to the collection of ions. However, to understand the role of the various different ions we have to consult the model results in order to determine the LC ions.

Before to run the model some considerations can be made on the transition frequencies and the nature of the underlying processes. For the transition frequency related to the conversion 
of species $p$ into $q$ the transition frequency $\mathscr{D}_{p, q}$ was introduced. In the case of radiative decay $\mathscr{D}_{p, l}=A_{p, l}$, being the probability of the radiative decay of $p$ to a lower level $l$. In the case of that 2 particles are involved we have $\mathscr{D}_{p, q}=n_{q} k_{p, q}^{(2)}$ where $n_{q}$ is the density of the particles colliding with $p$. For 3 -particles interaction we have $\mathscr{D}=n_{x} n_{y} k_{p, q}^{(3)}$.

A further classification can be made on the role of the excitation agent, the particle of type $x$ and $y$ as given above the expressions of the frequencies. We start with the electrons. These are found to be of main importance, especially there, where the active zone plasma is created. These endothermic processes, including excitation and ionization, are denoted by electron excitation kinetics (EEK). Once the radicals (the excited particles, ions) are formed, they can interchange there internal energy by means of charge or excitation transfer. The collection of these processes is denoted by heavy particle excitation kinetics (HEK). The excess of internal energy will be used to heat the gas. If the internal energy is transferred to the electrons we talk about MEK: mixed excitation kinetics. An example of a MEK process is Penning ionization. Finally we have the exothermal processes, the collection of recombination and deexcitation processes.

\section{Species and reactions included in the model}

The buffer gas atoms are uniformly distributed in time and space, i.e. the gas temperature, the gas pressure and density are independent on the spatial position. The gas temperature is assumed to be $1000 \mathrm{~K}$.

Table 1 lists the set of species for which the balance equations (equations (1) and (3)) were solved, together with the transport coefficients used in the model and the corresponding references.

\begin{tabular}{llll}
\hline Species $p$ & $\mu_{p} \times p\left(\right.$ Torr $\left.\mathrm{m}^{2} \mathrm{~V}^{-1} \mathrm{~s}^{-1}\right)$ & $D_{p} \times p\left(\right.$ Torr $\left.\mathrm{m}^{2} \mathrm{~s}^{-1}\right)$ & Ref. \\
\hline $\mathrm{e}$ & $f_{1}(\varepsilon)$ & equation (13) & {$[3]$} \\
$\mathrm{Ar}^{+}$ & $f_{2}(E / N)$ & equation $\mathbf{1 3})$ & {$[4]$} \\
$\mathrm{He}^{+}$ & $f_{3}(E / N)$ & equation & {$[4]$} \\
$\mathrm{Cu}^{+}$ & $f_{4}(E / N)$ & equation & {$[5]$} \\
$\mathrm{Cu}^{+*}$ & $f_{5}(E / N)$ & equation & {$[5]$} \\
$\mathrm{Ar}_{2}^{+}$ & $f_{6}(E / N)$ & equation & {$[4]$} \\
$\mathrm{He}_{2}^{+}$ & $f_{7}(E / N)$ & equation & {$[4]$} \\
$\left.\mathrm{Cu}^{+13}\right)$ & {$[6]$} \\
$\mathrm{Ar}^{*}$ & - & 13.45 & {$[7]$} \\
$\mathrm{He}^{*}$ & - & 20 & {$[7]$} \\
\hline
\end{tabular}

Table 1. The list of species for which the balance equation is solved, including the used transport coefficients and the corresponding references. The mobility coefficient for the electrons is specified as a function of the mean electron energy $f(\varepsilon)$. For the ionic species it is given as a function of the reduced electric field $f(E / N)$. The diffusion coefficients are deduced from the mobility using the Einstein relation 113 .

The $\mathrm{Cu}^{+*}$ in table 1 represents the excited ionic upper laser level, it is situated $24.5 \mathrm{eV}$ above the ground state of atomic $\mathrm{Cu}$.

The molecular ions are included as their influence could be of importance at the conditions under study. The intermediate states, the excited molecules, are not included in the model.

We assume that for the charged particles, the Einstein relation can be used to link the diffusion coefficients to the mobility:

$$
D_{p}=\frac{k_{\mathrm{B}} T_{p} \mu_{p}}{q_{p}}
$$

where $q_{p}$ is the elementary charge, and $T_{p}$ the particle temperature, corresponding to the energy of the random particle motion. 
For the ions the local field approximation is used. This assumes a direct relation between the particle energy distribution and the electric field, hence the transport coefficients are regarded as being functions of the reduced electric field. These functions can be found in literature as a result of experiments and classical theories [4,5]. The mobility of $\mathrm{He}^{+}$and $\mathrm{Ar}^{+}$ions in their pure parent gases as a function of the reduced electric field are adopted from [4]. From this the mobilities in a He-Ar gas mixture are calculated using Blanc's law [8].

For electrons, however, substantial deviations from the local field approximation can be expected for typical HCD conditions. Due to the small mass as compared to that of the heavy particles, electrons created in a high E-field region can easily be launched to regions where the E-field is small while keeping (part of) the initially gained high energy. Therefore, rather than using a dependence of the electron properties on $(E / N)$, we have to solve the electron energy balance. This gives the mean electron energy $\varepsilon(\mathbf{x})$ as a function of position. Subsequently the electron transport coefficients can be computed using the local $f(\varepsilon)$ values. The data are obtained from the free-ware Boltzmann equation solver BOLSIG + [3], which apart from the transport and reaction rate coeffcients gives insight in the electron energy disctribution function as well.

Since the individual metastable helium states participate in similar reactions with very similar rate coefficients [9], they are combined into one effective level, lying at $20.215 \mathrm{eV}$ above the ground level. The same applies to the Ar metastable levels: the metastable levels are combined into one effective level lying at $11.55 \mathrm{eV}$ above the argon ground state. Other excited states are not included in the model. In section 4.3] we will investigate what happens to the main plasma properties when the total excitation to all the excited levels is being set equal to ionization. By doing so, we in fact involve all the excited levels as stages in the ladder-climbing ionization process. This can be seen as giving the maximum in effective ionization. Excited states of sputtered $\mathrm{Cu}$ atoms are not taken into account since we do not expect that the (de)excitation of these levels will affect the electron energy and particle balance substantially.

Table 2 gives the complete set of reactions together with the reaction rate coefficients used in the model: the first 7 lines give the reactions induced by EEK, the group $8-11$ are HEK, the group $12-18$ are MEK. The next group of reactions from 19 to 22 are the recombination processes followed by the radiative process 23 .

As stated before, the buffer gas atoms, He and Ar in ground state, are assumed to be uniformly distributed in the discharge whereas the ground state copper atoms are produced by sputtering only. The atoms in ground state are ionized by electron collisions: direct ionization (reactions 3, 4 and 7) and excited to the lumped levels $\mathrm{Ar}^{*}$ and $\mathrm{He}^{*}$ (reactions 1 and 5). These levels can be converted into ions by means of electron collisions (reactions 2 and 6 ) and Penning ionization (reactions 12, 14, 15, 17 and 18). The HEK includes charge transfer reactions between the buffer gas ions and metal atoms in ground state (reactions 8 and 9). The processes of molecular ion formation include associative ionization (reactions 13 and 16) from the metastable levels and ion conversion (reactions 10 and 11), while the destruction process is dissociative recombination (reactions 20 and 21). The density of the excited $\mathrm{Cu}$ ionic level is mainly generated by charge transfer of $\mathrm{He}$ ions to $\mathrm{Cu}$ atoms (reaction 9) and decay spontaneously to the ground ionic level of $\mathrm{Cu}$ (reaction 23).

\section{Results and discussion}

The calculation results include the spatial distribution of the densities and fluxes of the species, as well as the rates of their production and loss processes. These are used to get volume averaged values of these quantities.

\subsection{Chemistry analysis}

Table 3 presents the reaction rates of each reaction averaged over the volume. From this table the following information can be deduced.

- The buffer gas atoms (Ar and He) are ionized mainly by electron impact; this can be due to direct ionization or stepwise ionization, that is ionization intermediated by excitation. From 


\begin{tabular}{rlcc}
\hline Nr. & Reaction & Rate coefficient, $k_{r}$ & References \\
\hline 1 & $\mathrm{e}+\mathrm{Ar} \rightarrow \mathrm{Ar}^{*}+\mathrm{e}$ & $k_{1}(\varepsilon) \mathrm{m}^{3} \mathrm{~s}^{-1}$ & {$[3]$} \\
2 & $\mathrm{e}+\mathrm{Ar}^{*} \rightarrow \mathrm{Ar}^{+}+2 \mathrm{e}$ & $k_{2}(\varepsilon) \mathrm{m}^{3} \mathrm{~s}^{-1}$ & {$[3]$} \\
3 & $\mathrm{e}+\mathrm{Ar} \rightarrow \mathrm{Ar}^{+}+2 \mathrm{e}$ & $k_{3}(\varepsilon) \mathrm{m}^{3} \mathrm{~s}^{-1}$ & {$[3]$} \\
4 & $\mathrm{e}+\mathrm{Cu} \rightarrow \mathrm{Cu}^{+}+2 \mathrm{e}$ & $k_{4}(\varepsilon) \mathrm{m}^{3} \mathrm{~s}^{-1}$ & {$[3]$} \\
5 & $\mathrm{e}+\mathrm{He} \rightarrow \mathrm{He}^{*}+\mathrm{e}$ & $k_{5}(\varepsilon) \mathrm{m}^{3} \mathrm{~s}^{-1}$ & {$[3]$} \\
6 & $\mathrm{e}+\mathrm{He}^{*} \rightarrow \mathrm{He}^{+}+2 \mathrm{e}$ & $k_{6}(\varepsilon) \mathrm{m}^{3} \mathrm{~s}^{-1}$ & {$[3]$} \\
7 & $\mathrm{e}+\mathrm{He} \rightarrow \mathrm{He}^{+}+2 \mathrm{e}$ & $k_{7}(\varepsilon) \mathrm{m}^{3} \mathrm{~s}^{-1}$ & {$[3]$} \\
8 & $\mathrm{Ar}^{+}+\mathrm{Cu} \rightarrow \mathrm{Ar}+\mathrm{Cu}^{+}$ & $3.16 \times 10^{-16} \mathrm{~m}^{3} \mathrm{~s}^{-1}$ & {$[10]$} \\
9 & $\mathrm{He}^{+}+\mathrm{Cu} \rightarrow \mathrm{He}+\mathrm{Cu}^{+*}$ & $1.0 \times 10^{-15} \mathrm{~m}^{3} \mathrm{~s}^{-1}$ & {$[10]$} \\
10 & $\mathrm{Ar}^{+}+2 \mathrm{Ar} \rightarrow \mathrm{Ar}_{2}^{+}+\mathrm{Ar}$ & $9.66 \times 10^{-56} \mathrm{~m}^{3} \mathrm{~s}^{-1}$ & {$[11]$} \\
11 & $\mathrm{He}^{+}+2 \mathrm{He} \rightarrow \mathrm{He}_{2}^{+}+\mathrm{He}$ & $6.8 \times 10^{-56} \mathrm{~m}^{3} \mathrm{~s}^{-1}$ & {$[12]$} \\
12 & $\mathrm{Ar}^{*}+\mathrm{Ar} \rightarrow \mathrm{Ar}+\mathrm{Ar}+\mathrm{e}$ & $1.17 \times 10^{-15} \mathrm{~m}^{3} \mathrm{~s}^{-1}$ & {$[11]$} \\
13 & $\mathrm{Ar}^{*}+\mathrm{Ar} \rightarrow \mathrm{Ar}+\mathrm{e}$ & $2.03 \times 10^{-15} \mathrm{~m}^{3} \mathrm{~s}^{-1}$ & {$[11]$} \\
14 & $\mathrm{Ar}^{*}+\mathrm{Cu} \rightarrow \mathrm{Ar}+\mathrm{Cu}+\mathrm{e}$ & $4.4 \times 10^{-16} \mathrm{~m}^{3} \mathrm{~s}^{-1}$ & {$[10]$} \\
15 & $\mathrm{He}^{*}+\mathrm{He} * \mathrm{He}+\mathrm{He}+\mathrm{e}$ & $0.87 \times 10^{-15} \mathrm{~m}^{3} \mathrm{~s}^{-1}$ & {$[12]$} \\
16 & $\mathrm{He}^{*}+\mathrm{He} \rightarrow \mathrm{He}++\mathrm{e}$ & $1.5 \times 10^{-15} \mathrm{~m}^{3} \mathrm{~s}^{-1}$ & {$[12]$} \\
17 & $\mathrm{He}^{*}+\mathrm{Ar} \rightarrow \mathrm{He}+\mathrm{Ar}+\mathrm{e}$ & $1.68 \times 10^{-16} \mathrm{~m}^{3} \mathrm{~s}^{-1}$ & {$[10]$} \\
18 & $\mathrm{He}^{*}+\mathrm{Cu} \rightarrow \mathrm{He}+\mathrm{Cu}++\mathrm{e}$ & $9.48 \times 10^{-16} \mathrm{~m}^{3} \mathrm{~s}^{-1}$ & {$[10]$} \\
19 & $\mathrm{Ar}^{+}+2 \mathrm{e} \rightarrow \mathrm{Ar}+\mathrm{e}$ & $k_{8}(\varepsilon) \mathrm{m}^{3} \mathrm{~s}^{-1}$ & {$[3]$} \\
20 & $\mathrm{Ar}_{2}^{+}+\mathrm{e} \rightarrow \mathrm{Ar}^{*}+\mathrm{Ar}$ & $k_{9}(\varepsilon) \mathrm{m}^{3} \mathrm{~s}^{-1}$ & {$[11]$} \\
21 & $\mathrm{He}_{2}^{+}+\mathrm{e} \rightarrow \mathrm{He}^{*}+\mathrm{He}$ & $k_{10}(\varepsilon) \mathrm{m}^{3} \mathrm{~s}^{-1}$ & {$[12]$} \\
22 & $\mathrm{He}^{+}+2 \mathrm{e} \rightarrow \mathrm{He}+\mathrm{e}$ & $k_{11}(\varepsilon) \mathrm{m}^{3} \mathrm{~s}^{-1}$ & {$[3]$} \\
23 & $\mathrm{Cu}^{+*} \rightarrow \mathrm{Cu}^{+}+h \nu$ & $0.23 \times 10^{9} \mathrm{~s}^{-1}$ & {$[13]$} \\
\hline
\end{tabular}

Table 2. Reaction rate coefficients used in the model. The electron impact reactions are specified as a function of the mean electron energy $k_{r}(\varepsilon)$.

the table it can be seen that the rates of excitation and ionization of Ar are much larger than those of He. This is because the ionization (excitation) potential of Ar is lower (24.58 versus $15.76 \mathrm{eV}$ for ionization and 20.215 versus $11.62 \mathrm{eV}$ for excitation). This implies that the $\mathrm{Ar}$ ion is the main ionic species in the discharge in spite of the fact that Ar consists of only $5 \%$ of the gas mixture. Comparing the electron excitation kinetics EEK with HEK shows that the electron impact processes have the highest rates. This makes that the system can be seen as an ionising EEK plasma.

- The rates of the backward processes, atomic recombination (reactions 19 and 21), are in the order of $10^{16} \mathrm{~m}^{-3} \mathrm{~s}^{-1}$ for $\mathrm{He}^{+}$and $\mathrm{Ar}^{+}$. Since they are 8 orders of magnitude lower than the forward process, it is obvious that they can be neglected.

- The excited ion level $\mathrm{Cu}^{+*}$ is only produced by charge transfer with He atoms (reaction 9) and decay spontaneously to $\mathrm{Cu}^{+}$(reaction 23). It can be seen from table 3 that the rates of these two reactions are the same, meaning that the criteria (11) holds for $\mathrm{Cu}^{+*}$. This confirms what was found before: $\mathrm{Cu}^{+*}$ is a LC species.

- The production processes of molecular ions include ion conversion (reactions 10 and 11) and associative ionization (reactions 13 and 16). The calculated density of $\mathrm{He}_{2}^{+}$is less than 1000 smaller than the density of $\mathrm{He}^{+}$. The same holds more or less for the ratio $\mathrm{Ar}_{2}^{+} / \mathrm{Ar}^{+}$.

For $\mathrm{Ar}_{2}^{+}$the dominant production process in the associative ionization with a rate in the order of $10^{23} \mathrm{~m}^{-3} \mathrm{~s}^{-1}$ whereas the ion conversion rate is in order of $10^{19} \mathrm{~m}^{-3} \mathrm{~s}^{-1}$.

Due to the low densities of $\mathrm{He}_{2}^{+}$and $\mathrm{Ar}_{2}^{+}$and consequently low fluxes to the cathode wall, the contribution of the molecular ions to sputtering can be neglected. 


\begin{tabular}{|c|c|c|}
\hline Nr. & Reaction & Rate $\left(\mathrm{m}^{-3} \mathrm{~s}^{-1}\right)$ \\
\hline 1 & $\mathrm{e}+\mathrm{Ar} \rightarrow \mathrm{Ar}^{*}+\mathrm{e}$ & $7.75 \times 10^{24}$ \\
\hline 2 & $\mathrm{e}+\mathrm{Ar}^{*} \rightarrow \mathrm{Ar}^{+}+2 \mathrm{e}$ & $6.77 \times 10^{24}$ \\
\hline 3 & $\mathrm{e}+\mathrm{Ar} \rightarrow \mathrm{Ar}^{+}+2 \mathrm{e}$ & $2.16 \times 10^{24}$ \\
\hline 4 & $\mathrm{e}+\mathrm{Cu} \rightarrow \mathrm{Cu}^{+}+2 \mathrm{e}$ & $1.42 \times 10^{24}$ \\
\hline 5 & $\mathrm{e}+\mathrm{He} \rightarrow \mathrm{He}^{*}+\mathrm{e}$ & $4.46 \times 10^{23}$ \\
\hline 6 & $\mathrm{e}+\mathrm{He}^{*} \rightarrow \mathrm{He}^{+}+2 \mathrm{e}$ & $1.73 \times 10^{23}$ \\
\hline 7 & $\mathrm{e}+\mathrm{He} \rightarrow \mathrm{He}^{+}+2 \mathrm{e}$ & $3.71 \times 10^{22}$ \\
\hline 8 & $\mathrm{Ar}^{+}+\mathrm{Cu} \rightarrow \mathrm{Ar}+\mathrm{Cu}^{+}$ & $4.86 \times 10^{22}$ \\
\hline 9 & $\mathrm{He}^{+}+\mathrm{Cu} \rightarrow \mathrm{He}+\mathrm{Cu}^{+*}$ & $3.44 \times 10^{21}$ \\
\hline 10 & $\mathrm{Ar}^{+}+2 \mathrm{Ar} \rightarrow \mathrm{Ar}_{2}^{+}+\mathrm{Ar}$ & $4.72 \times 10^{19}$ \\
\hline 11 & $\mathrm{He}^{+}+2 \mathrm{He} \rightarrow \mathrm{He}_{2}^{+}+\mathrm{He}$ & $3.35 \times 10^{20}$ \\
\hline 12 & $\mathrm{Ar}^{*}+\mathrm{Ar}^{*} \rightarrow \mathrm{Ar}^{+}+\mathrm{e}+\mathrm{Ar}$ & $1.3 \times 10^{23}$ \\
\hline 13 & $\mathrm{Ar}^{*}+\mathrm{Ar}^{*} \rightarrow \mathrm{Ar}_{2}^{+}+\mathrm{e}$ & $2.2 \times 10^{23}$ \\
\hline 14 & $\mathrm{Ar}^{*}+\mathrm{Cu} \rightarrow \mathrm{e}+\mathrm{Ar}+\mathrm{Cu}^{+}$ & $1.32 \times 10^{23}$ \\
\hline 15 & $\mathrm{He}^{*}+\mathrm{He}^{*} \rightarrow \mathrm{e}+\mathrm{He}+\mathrm{He}^{+}$ & $6.73 \times 10^{19}$ \\
\hline 16 & $\mathrm{He}^{*}+\mathrm{He}^{*} \rightarrow \mathrm{He}_{2}^{+}+\mathrm{e}$ & $1.16 \times 10^{20}$ \\
\hline 17 & $\mathrm{He}^{*}+\mathrm{Ar} \rightarrow \mathrm{He}+\mathrm{Ar}^{+}+\mathrm{e}$ & $2.61 \times 10^{23}$ \\
\hline 18 & $\mathrm{He}^{*}+\mathrm{Cu} \rightarrow \mathrm{e}+\mathrm{He}+\mathrm{Cu}^{+}$ & $8.59 \times 10^{21}$ \\
\hline 19 & $\mathrm{Ar}^{+}+\mathrm{e}+\mathrm{e} \rightarrow \mathrm{Ar}+\mathrm{e}$ & $1.34 \times 10^{16}$ \\
\hline 20 & $\mathrm{Ar}_{2}^{+}+\mathrm{e} \rightarrow \mathrm{Ar}^{*}+\mathrm{Ar}$ & $1.04 \times 10^{23}$ \\
\hline 21 & $\mathrm{He}^{+}+\mathrm{e}+\mathrm{e} \rightarrow \mathrm{He}+\mathrm{e}$ & $6.04 \times 10^{16}$ \\
\hline 22 & $\mathrm{He}_{2}^{+}+\mathrm{e} \rightarrow \mathrm{He}^{*}+\mathrm{He}$ & $2.56 \times 10^{19}$ \\
\hline 23 & $\mathrm{Cu}^{+*} \rightarrow \mathrm{Cu}^{+}+\mathrm{h} \nu$ & $3.2 \times 10^{21}$ \\
\hline
\end{tabular}

Table 3. Calculated reaction rates averaged over the volume.

Table 4 presents the calculated contribution in percents of every reaction to the production/destruction rate for each relevant species. The ratio between the production and destruction rate $\mathscr{P}_{p} / \mathscr{L}_{p}$ is shown as well. This helps to determine the locality/non-locality of the species.

- Electrons. The most important electron production processes are the direct $(\sim 20 \%)$, and the stepwise ionization $(\sim 60 \%)$ of Ar. Ionization of $\mathrm{Cu}$ contributes with about $13 \%$. This is a substantial contribution especially in view of the fact that the $\mathrm{Cu}$ atom density forms less than $0.1 \%$ of the gas mixture. The reason lies again in the ionization potential; this is lower than that of Ar and He. The Penning processes contribute with a only a few percents to the creation of electrons.

From table 4 can be seen that the loss rate of electrons is two orders of magnitude lower than the production rate, so that $\mathscr{P}_{p} / \mathscr{L}_{p} \gg 1$, As it was expected the electrons are TS species.

- Excited species. The Ar excited states are produced mainly (98.7\%) by electron impact excitation of the ground state and are predominantly lost by ionization (89.1\%). Only a few percents is lost by MEK processes (reactions 12, 13 and 14).

For He* the production is $100 \%$ due to electron impact excitation of the ground level. The Penning process that transforms Ar ground atoms into $\mathrm{Ar}^{+}$(reaction 17) appears to be the dominant loss process of $\mathrm{He}^{*}(58.9 \%)$; another substantial destruction channel is that of ionization $(39 \%)$.

From table 4 it can be seen that the excited gas species are completely determined by the reactions occurring in the discharge: $\mathscr{P} / \mathscr{L}=1$, which completely agrees with the considerations given in section 2 However, despite of the locality, they give an important contribution to the (stepwise) ionization. Around $80 \%$ of the He ions are produced by stepwise ionization versus $20 \%$ by direct ionization. For $\mathrm{Ar}^{+}$the ratio is more or less the same. As 


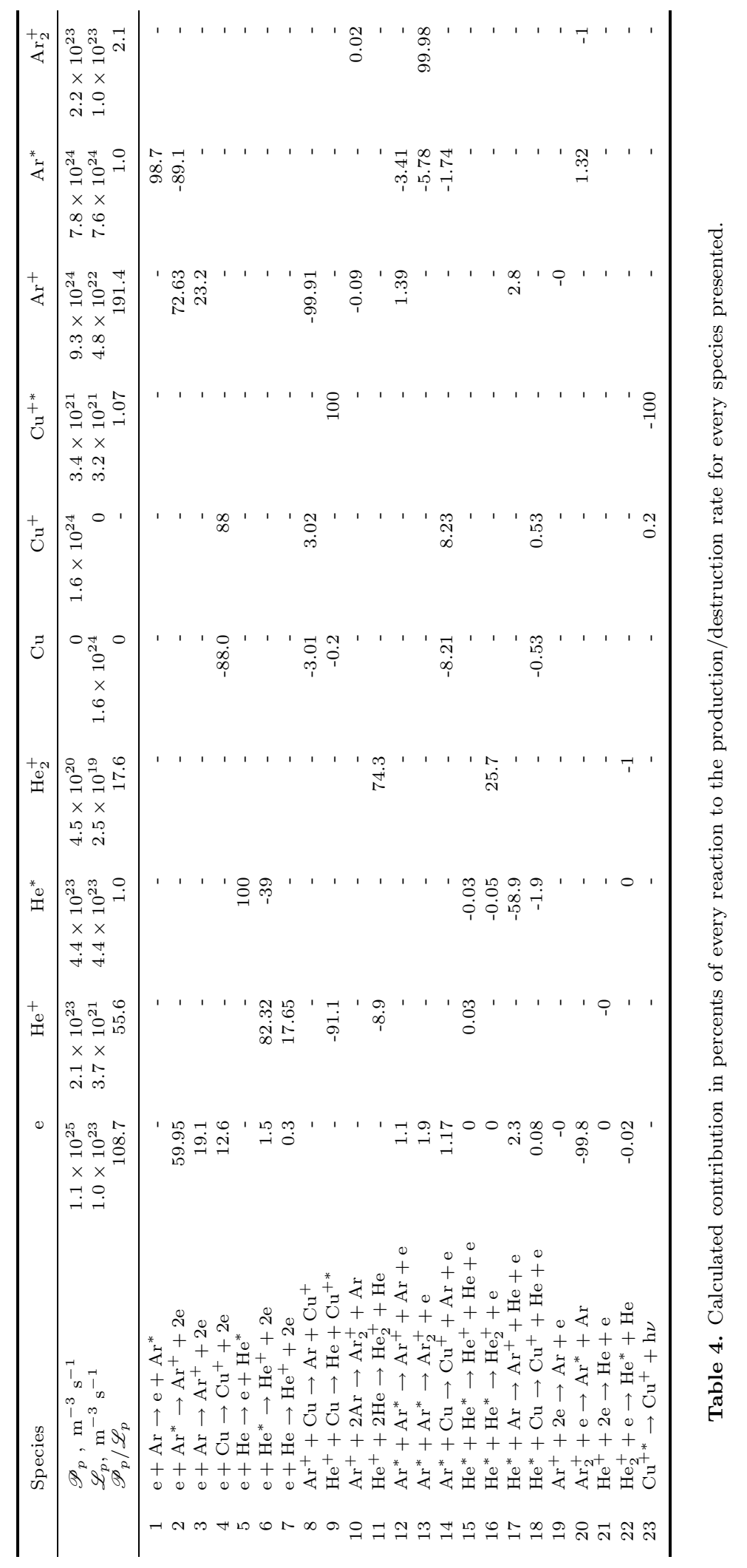


discussed in section 2 for LC species it is not necessary to solve the transport equation (3). This speed up the calculations. Concluding we may state that there is no need to employ the particle balance in full glory for the computation of the density of excited gas species $\mathrm{Ar}^{*}$ and $\mathrm{He}^{*}$. As LC species local production/destruction balances suffice. However, the species can not be removed since they serve as important intermediate stages in the ionization processes.

- Molecular ions. For $\mathrm{He}_{2}^{+}$the production is mainly $(\sim 74 \%)$ due to ion conversion process and associative ionization $(\sim 26 \%)$. They are lost by dissociative recombination. The calculated $\mathrm{He}_{2}^{+}$density is in the order of $10^{14} \mathrm{~m}^{-3}$. From table 4 it can be seen that these processes give minor contributions for the production/destruction of the other species. The $\mathrm{He}_{2}^{+}$are unprofitable in the discharge.

For $\mathrm{Ar}_{2}^{+}$the main production process $(99.9 \%)$ is associative ionization (reaction 20). From table 4 it can be seen that this process have minor effect to the production/destruction of other species. The contribution of the ion conversion process to the production of $\mathrm{Ar}_{2}^{+}$is negligible $(0.02 \%)$; moreover this process does not contribute to the production/destruction of any other species, hence it can be neglected.

The dissociative recombination of $\mathrm{Ar}_{2}^{+}$appears to be the main loss process of electrons. However, the calculated rate for this process is two orders of magnitude lower than the total ionization. Excluding this process will not affect the electron density because the ionization processes are the dominant processes in the discharge.

The ratio $\mathscr{P}_{p} / \mathscr{L}_{p}$ of $\mathrm{He}_{2}^{+}$is $\sim 18$. That means the $\mathrm{He}_{2}^{+}$is almost transport driven. This is not so surprising since $\mathrm{He}_{2}^{+}$has a high mobility. The ratio between the production and destruction processes for $\mathrm{Ar}_{2}^{+}$is 2 and indicates that the density of $\mathrm{Ar}_{2}^{+}$is predominantly determined by the local reactions in the discharge. At the condition under study, the locality of the $\mathrm{Ar}_{2}^{+}$allows even to ignore the presence of the Ar molecular ions; the density is small and the contribution to production or destruction for other species is negligible. The elimination of the molecular ions is discussed in next subsection.

\subsection{Molecular ions}

To analyse the possible consequences of ignoring molecular ions and the related reactions, two models were constructed: the first one is equipped with the complete set of species and reactions as shown in the tables $\mathbf{1 1}$ and $\mathbf{2}$ the second model is a simplified model with reduced chemistry according to the observations above, i.e. excluding molecular ions and the relevant reactions, and atomic recombination processes. For both models the power is set to $300 \mathrm{~W}$ while the pressure is chosen to be $2.3 \mathrm{kPa}$.

Figure 11 compares the electron, $\mathrm{He}^{+}$and $\mathrm{Ar}^{+}$densities obtained by the two models. It is clear that the presence of molecular ions does not affect the density distribution of other species behaviour in the discharge. They can be excluded in the reduced chemistry model.

\subsection{Ladder climbing}

In section 2 was shown, and in subsection 4.1 confirmed by the model, that the metastable levels are LC species. They are created predominantly by excitation and immediately lost by ionisation, contributing greatly to the ionization degree. However, in practice EEK processes to higher excited levels can also contribute to the ionization degree; the so-called ladder-climbing processes might be of importance [14]. In order to check the importance/unimportance of these processes, we will construct another model and again compare the results with the complete model.

From the same considerations as described above, we can expect that species in all the possible excited states are LC species and that they, after being created, are immediately ionized. So that we can consider that the total excitation rate equals to the total ionization rate. With this assumption, the reduced chemistry model can be further simplified excluding completely the metastable levels and hence omitting the intermediate step of excitation. So, the reactions left in the reduced chemistry model are presented in table 5 The EEK rate coefficients as a function of 


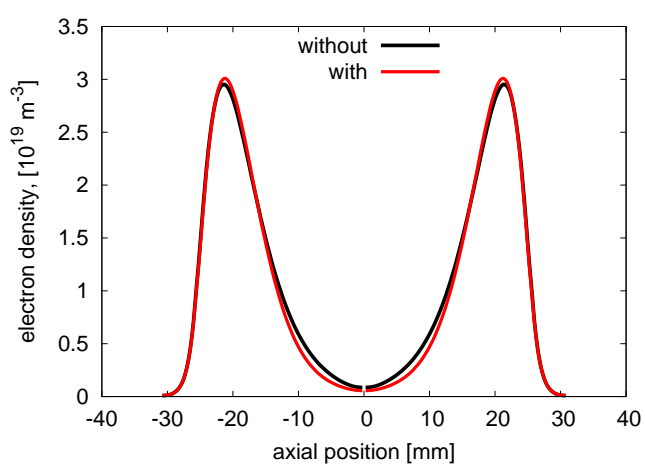

(a) Electron density

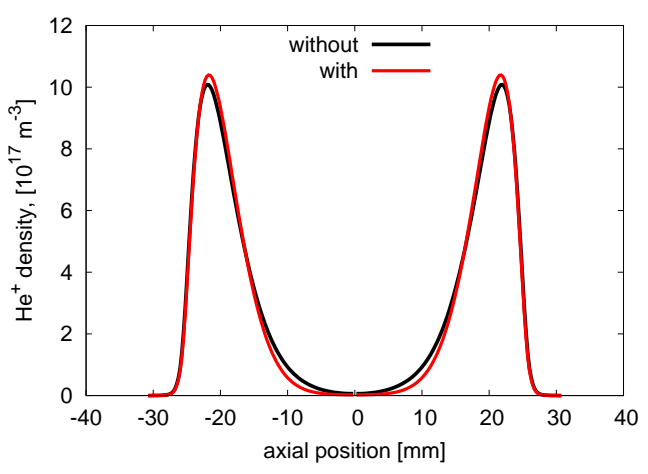

(b) He ion density

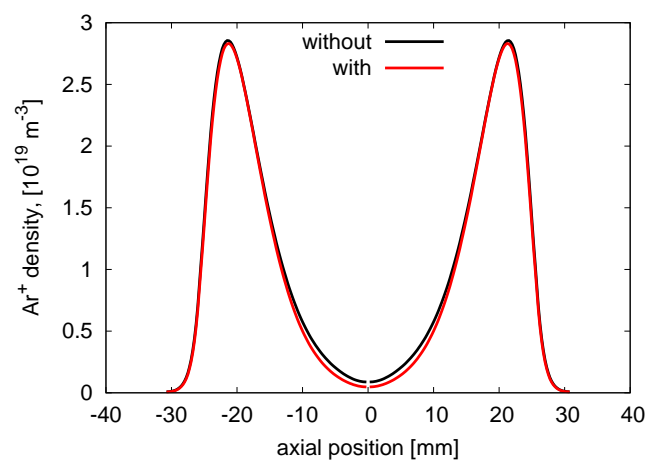

(c) Ar ion density

Figure 1. Comparison of the axial density profiles of two models with and without molecular ions.

\begin{tabular}{rlcc}
\hline Nr. & Reaction & Rate coefficient, $k_{r}\left(\mathrm{~m}^{3} \mathrm{~s}^{-1}\right)$ & References \\
\hline 1 & $\mathrm{Ar}+\mathrm{e} \rightarrow \mathrm{Ar}^{+}+2 \mathrm{e}$ & $k_{\text {exc }}(\varepsilon)$ & {$[3]$} \\
2 & $\mathrm{Cu}+\mathrm{e} \rightarrow \mathrm{Cu}^{+}+2 \mathrm{e}$ & $k_{\text {exc }}(\varepsilon)$ & {$[3]$} \\
3 & $\mathrm{He}+\mathrm{e} \rightarrow \mathrm{He}^{+}+2 \mathrm{e}$ & $k_{\text {exc }}(\varepsilon)$ & {$[3]$} \\
4 & $\mathrm{Ar}^{+}+\mathrm{Cu} \rightarrow \mathrm{Ar}+\mathrm{Cu}^{+}$ & $3.16 \times 10^{-16}$ & {$[10]$} \\
5 & $\mathrm{He}^{+}+\mathrm{Cu} \rightarrow \mathrm{He}+\mathrm{Cu}^{+*}$ & $1.0 \times 10^{-15}$ & {$[10]$} \\
\hline
\end{tabular}

Table 5. Reaction rate coefficients used in the simple model.

the mean electron energy are replaced with the rate coefficient for the total excitation. From the HEK processes we have left only the charge transfer processes of metal atoms with gas ions.

Table [6] presents the densities of the active species, averaged over the volume in comparison with the complete model. Table [6] shows small differences in the averaged values of the densities. The densities are slightly higher for the simple model. The densities of electrons and Ar ions are higher for the simplified model, while the density of He ions is lower. The reason is that the increased ionization in the simplified model results in lower electron energy. The ionization rate will be higher for species with lower ionization potential. For $\mathrm{Cu}$ ions the difference is more pronounced also because the increased Ar ion density results in increased sputtering, hence the density of $\mathrm{Cu}$ atoms in the discharge. Nevertheless, there is not considerable change in the species densities.

Figure 2 presents the axial profiles of the electron density and the ion densities. It can be seen 


\begin{tabular}{lll}
\hline Density, $\mathrm{m}^{-3}$ & Complete Model & Simple Model \\
\hline $\mathrm{e}$ & $7.85 \times 10^{18}$ & $9.14 \times 10^{18}$ \\
$\mathrm{Ar}^{+}$ & $7.29 \times 10^{18}$ & $8.81 \times 10^{18}$ \\
$\mathrm{He}^{+}$ & $2.03 \times 10^{17}$ & $1.76 \times 10^{17}$ \\
$\mathrm{Cu}^{+}$ & $1.68 \times 10^{17}$ & $2.48 \times 10^{17}$ \\
$\mathrm{Cu}$ & $3.58 \times 10^{19}$ & $4.52 \times 10^{19}$ \\
\hline
\end{tabular}

Table 6. Species densities averaged over the volume. Comparison between the complete model and the simple one.

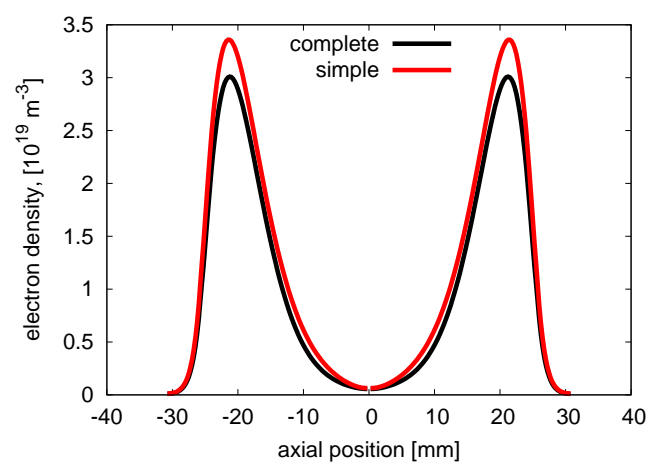

(a) Electron density

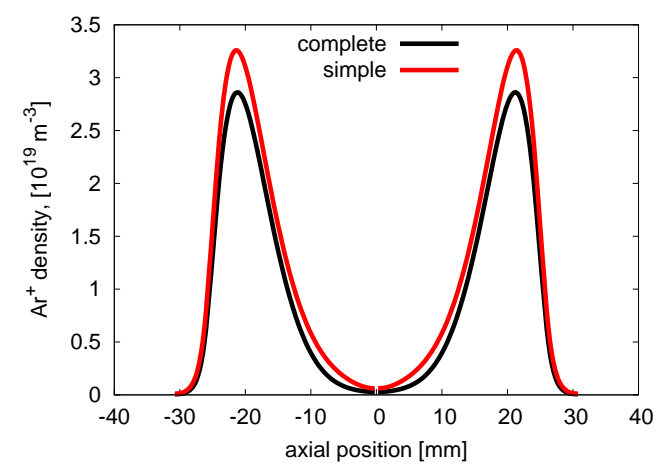

(b) Ar ion density

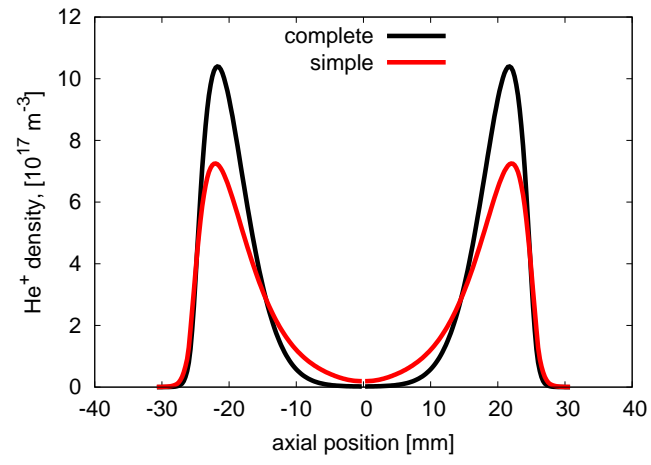

(c) He ion density

Figure 2. Comparison of the axial density profiles of two models: the complete model and the simpler one (without excited states).

that the axial profiles of the main charged particles remains more or less the same. The difference lies in the regions where the plasma density is highest. Apart from the slightly changes in the maximum values of the densities, the spatial density distribution is not changed significantly. We have to conclude that the contribution to the ionization degree due to ladder-climbing is negligible at the conditions under study.

\section{Conclusions}

In a search of a reduced chemistry module for the description of the plasma behaviour in a HCD with a He-Ar-Cu mixture, estimative calculations and numerical simulations are performed. This reduced chemistry model aims to construct a lean and reliable model that can be used as part of a design tool of HCD lasers. 
Analysing the various species and their behaviour we determined the species that are not essential for the discharge, meaning that the presence of these species does not affect the main plasma properties (spatial values of the electron density and mean energy) so that they are not essential for the plasma application.

Based on the estimative calculations, numerical simulations and the post processing of the modelling results, we can summarise the following:

- The HCD plasma is characterised as an ionising electron excitation kinetics (EEK) plasma; the main ionic species is $\mathrm{Ar}^{+}$, despite the fact that Ar constitute only $5 \%$ of the gas mixture.

- Being an ionising plasma, the atomic recombination processes can be neglected.

- Electrons and ions are transport sensitive (TS) species, while excited species (atom and ion) are local chemistry (LC) species. This observation results in simplification of the continuity equations for the LC species as the transport term is zero.

- The influence of the molecular ions is negligible at the conditions under study. Although in many cases the molecular ions could appear as an important recombination chanel, it was shown that for the conditions under study all the recombination processes can be neglected with respect to the ionization processes.

- The contribution to the ionization degree due to ladder-climbing is negligible at the conditions under study.

Taking the statements above into considerations, enables to construct a lean and reliable model, without affecting the main plasma properties and without loss of accuracy. With such a chemical lean model it is than possible to find the best of the geometrical construction, the anode cathode patterns, the current settings, the fill-chemistry, etc. The technique developed in this study can be applied to glow discharges in general and to ionising electron excitation kinetics (EEK) plasmas.

\section{Acknowledgments}

This work was done as part of "Physical chemistry of plasma-surface interaction" (PSI) in the framework of the Belgian federal programme "Interuniversity Attraction Poles" (IAP).

The authors acknowledge the support of the Dutch National Programme BSIK, in the ICT project BRICKS, theme MSV1 and the support of the National Science Fund of Bulgaria, project DO $02-274 / 2008$.

\section{References}

[1] Mihailova D, Grozeva M, Hagelaar G, van Dijk J, Brok W and van der Mullen J 2008 J Phys D Appl Phys 41245202

[2] Mihailova D, van Dijk J, Grozeva M, Hagelaar G and van der Mullen J 2010 J Phys D Appl Phys 43145203

[3] Hagelaar G and Pitchford L 2005 Plasma Sources Sci Technol 14 722-733

[4] Ellis H, Pai R, McDaniel E, Mason E and Viehland L 1976 At Data and Nucl Data Tables 17177

[5] McDaniel E 1964 Collision phenomena in ionized gases (New York: Wiley \& Sons)

[6] Sekido H, Kondo T, Kono A and Goto T 1993 J Phys D Appl Phys 261414

[7] Grigoriev I, Meilikhov E and Radzig A 1997 Handbook of physical quantities (CRC Press, London)

[8] Blanc A 1908 Journal de Physique Theorique et Appliquee 7825

[9] Browne P and Dunn M 1973 J Phys B At Mol Opt Phys 61103

[10] Arslanbekov R, Tobin R and Kudryatsev A 1997 J Appl Phys 81554

[11] Klucharev A and Vujnovic V 1990 Physics Reports 18555

[12] Deloche R, Monchicourt P, Cheret M and Lambert F 1976 Phys Rev A 131140

[13] Theodosiou C 1986 J Opt Soc Am B 31107

[14] van der Mullen J 1990 Physics Reports 191 109-220 